\title{
Improving Ultra Wide Band Imaging using a Metamaterial Slab
}

\author{
Ahmed M. D. E. Hassanein ${ }^{1}$, Alonso Corona Chávez ${ }^{2}$
}

\begin{abstract}
The quality of tomographic images formed from diffracting waves deteriorates due to the limitations in capturing evanescent waves. The waves carry detailed information about the body being imaged. Metamaterials are being recently used to amplify and focus evanescent waves. Here, the physical characteristics of a hyperlens made out of a silver metamaterial slab are studied. A description of the equations for wave propagation inside the metamaterial is shown. The dependence of the cutoff frequency of a hyperlens on the thickness of the slab is discussed. Suitable physical dimensions of a hyperlens are proposed for cross sectional imaging of objects imitating the characteristics of human body. Simulations are done to test the validity of the proposed solution.
\end{abstract}

\section{Index Terms-Tomography, Evanescent Waves, Metamaterials, Hyperlens, Perfect Imaging.}

\section{INTRODUCTION}

Microwave imaging is being investigated widely for medical and process tomography. The formation of tomographic images from ultra wideband radio signals is often problematic due to limitations arising from diffraction and refraction as well as attenuation. Many approaches to diffraction tomography rely on complex iterative approaches to solve the inverse problem of determining the object from scattered signals, or on some assumed foreknowledge of the dielectric of the environment / object being probed. The virtual confocal microscope method has been proven theoretically [1] and cross sectional imaging has been demonstrated [1] without either of these problems. The foundations of this method have been the topic of several research investigations including but not limited to the thesis [2]. The method begins with a measurement of the wave fronts leaving the target and uses these in conjunction with a synthetic optical system to form a confocal image by computing the wave propagation through the effective optics to form the image [1].

The aim of this paper is to enhance the resolution limit of the optical system. We propose constructing a hyper lens through a continuous media that would allow the user to fully control the field scattered from the imaged object and enable us to significantly enhance the resolution imposed by the diffraction limit [3]. Starting with the design process, suitable frequency and size of a hyper lens will be established. A one dimensional super lens will be tested to evaluate its performance and compare images with object being imaged.

\section{PRoblem AND SOLUTION}

Problems with resolution when imaging using Ultra Wide Band waves were discussed in several papers $[4,5,6]$. If a wave is incident upon a boundary between two media with angle greater than the critical angle, evanescent waves are generated or total internal reflection happens. Critical angle is defined

Manuscript received January 31, 2014. (1) The author is within the Systems and Information Department, National Research Centre (NRC), Dokki, Cairo, Egypt. email: ahmed22@aucegypt.edu, Tel: 012-78996660. (2) The author is within the Department of Electronica, Instituto Nacional De Astrofisica, Optica Y Electronica (INAOE), Tonantzintla, Puebla, Mexico. This work was supported by a CONACYT-TWAS Postdoctoral Fellowship (FR number: 3240255079) covered by The National Council on Science and Technology (CONACYT) and The Academy of Sciences for the Developing World (TWAS). 
using Snell's Law of refraction [7]. If a wave is incident on any object, evanescent waves are created as well. The frequency of the scattered evanescent waves is dependent on the shape of the object and the details in it. The more the details the higher the frequency components scattered from the object. Evanescent waves decay exponentially and can't be captured by antennas. The amplitude of a received signal is attenuated and so the quality of calculated images decreases.

Due to the diffraction limit [7], an object with dimensions less than half the wavelength used cannot be imaged. Detailed information about the object being imaged is held by the evanescent waves which can't be captured due to the above mentioned reason. A metamaterial slab can be used to make the capturing of those waves possible and so the ability to produce a perfect image for an object is possible. In this section, the equations for calcculating the electric field inside free space and a slab made out of a metamaterail are given. Then, the effect of propagating waves in either free space of the slab is studied.

\section{A. Equations Describing Electric Field in Different Media}

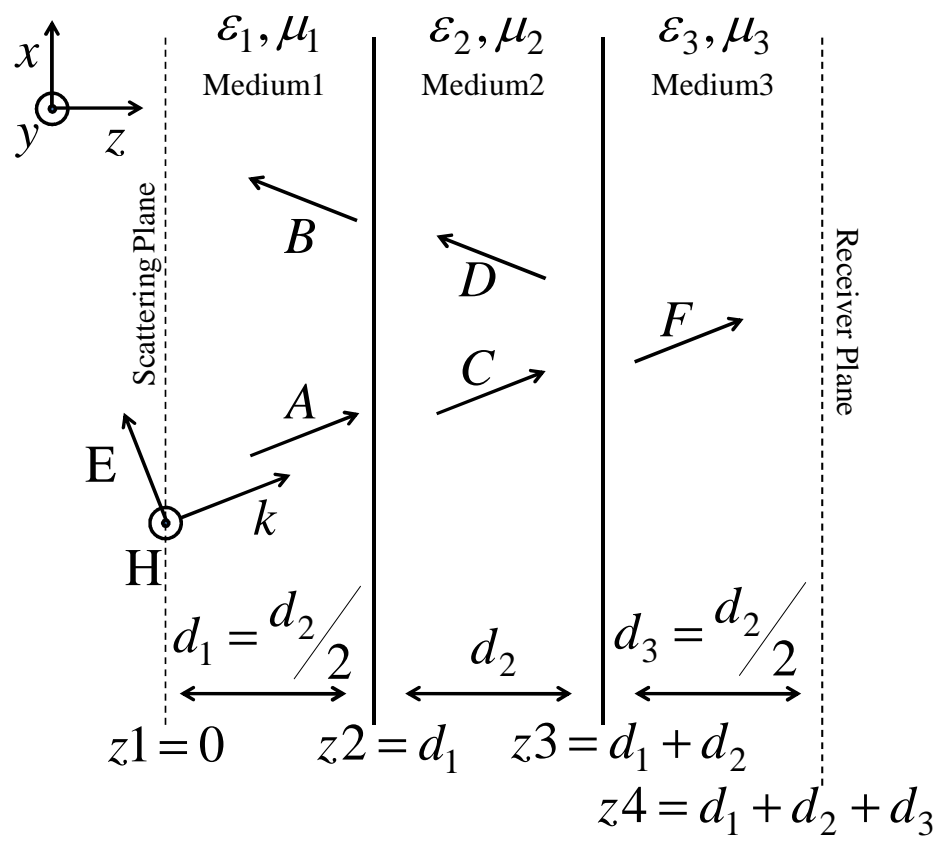

Figure 1: A sketch not to scale is shown of a superlens (medium2) inserted in free space (medium1,3). The permittivity and permuability of the different media are shown with thichnesses $d_{1}, d_{2}$ and $d_{3}$. A TM wave is scattedred at the scattering plane and received at the receiver plane.

The slab is represented in fig. 1 by medium2. The slab is inserted in free space which is represented by medium1 and 3. For a TM electromagnetic wave propagating through the three media, the $x$ component of the electric field in medium $1 \mathrm{E}_{x 1}$ is [8]:

$\mathrm{E}_{x 1}(x, z)=\sum_{k_{x 1}} \frac{k_{z 1}}{\omega \varepsilon_{1}}\left(A e^{-j k_{z 1} z}-B e^{j k_{z 1} z}\right) e^{-j k_{x 1} x}$

where $k_{z 1}$ is the $z$ component of the wave vector $k, \omega$ is the angular frequency of the waves used, $\varepsilon_{1}$ is the permittivity of medium $1, k_{x 1}$ is the $x$ component of the wave vector in medium $1, A$ is the amplitude of the incident waves on the front surface of the slab and $B$ is the amplitude of the reflected waves from the front surface of the slab (refer to fig. 1). The relation between the $z$-component and the $x$-component of the of the wave vector is:

$\sqrt{k_{z}^{2}+k_{x}^{2}}=k_{0}^{2}$

where $k_{0}^{2}=\omega^{2} \varepsilon \mu$.

The $x$ component of the electric field in medium $2 \mathrm{E}_{x 2}$ is [8]: 
$\mathrm{E}_{x 2}(x, z)=\sum_{k_{x 2}} \frac{k_{z 2}}{\omega \varepsilon_{2}}\left(C e^{-j k_{z 2} z}-D e^{j k_{z 2} z}\right) e^{-j k_{x 2} x}$

where $k_{z 2}$ is the $z$ component of the wave vector in medium2, $\varepsilon_{2}$ is the permittivity of medium2, $k_{x 2}$ is the $x$ component of the wave vector in medium2, $C$ is the amplitude of the waves transmitted from medium 1 to medium 2 and $D$ is the amplitude of the reflected waves from the rear surface of the slab.

The $x$ component of the electric field in medium $3 \mathrm{E}_{x 3}$ is [8]:

$\mathrm{E}_{x 3}(x, z)=\sum_{k_{x 3}} \frac{k_{z 3}}{\omega \varepsilon_{3}}\left(F e^{-j k_{z 3} z}\right) e^{-j k_{x 3} x}$

where $k_{z 3}$ is the $z$ component of the wave vector in medium3, $\varepsilon_{3}$ is the permittivity of medium3, $k_{x 3}$ is the $x$ component of the wave vector in medium3 and $F$ is the amplitude of the waves transmitted from medium 2 to medium3. For any kind of object that is paced at the scattering plane, the amplitude of the electric field scattered from it changes as it passes through the different media as shown in the next subsection.

\section{B. Propagation of Electric Field in Different Media}

Using Eq. 1,3,4, the amplitude of the propagating electric field is plotted across the three media shown in fig. 1 .

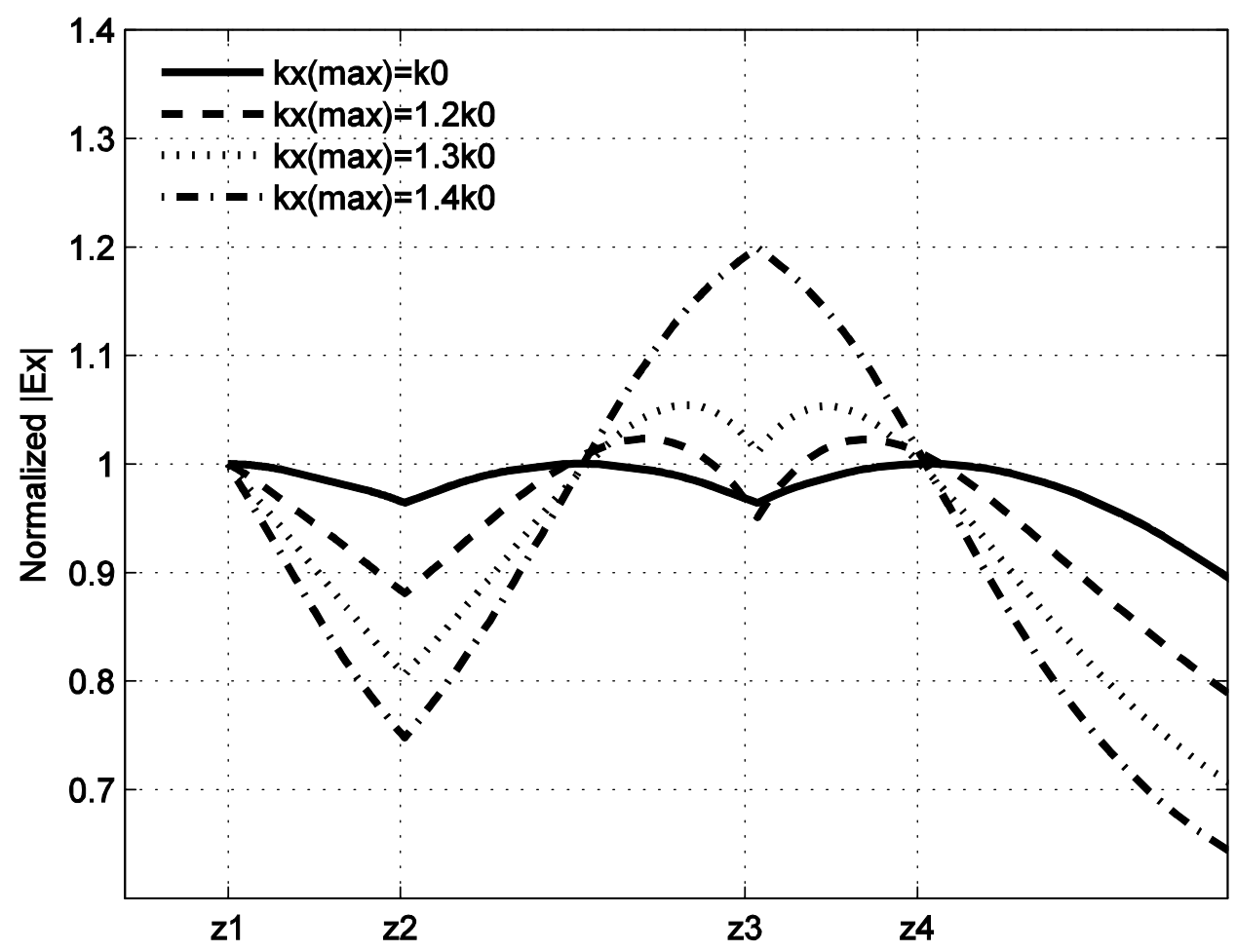

Figure 2: The plot shows the maximum amplitude of the electric field after passing through the three media which are shown in fig.1 [8].

In fig. 2, we can see four lines representing the maximum amplitude of the electric field as it propagates through the slab and free space. The solid line represents the summation of all frequencies that satisfy the range $0<k_{x}<k_{0}$. A maximum is seen in the middle of the slab. The dashed line represents the summation of frequencies satisfying the range $0<k_{x}<1.2 k_{0}$. While the dotted line includes all frequencies satisfying the range $0<k_{x}<1.3 k_{0}$. The dash-dotted line includes all frequencies satisfying the range $0<k_{x}<1.4 k_{0}$. As more evanescent components are added the maximum inside 
the slab moves to the rear surface of the slab. In all cases, an image that has the same amplitude as the object placed at $z 1$ is seen at $z 4$. After $z 4$, all cases decay rapidly.

\section{Simulation Results}

In this section, we study, through simulation, the possibility of imaging an object that is ten times smaller than the wavelength used using a metamaterial slab. The object function at the scattering line is defined. Spacial frequencies are defined for the object function by taking the fourier transform of it. The total tarnsfer function of the media in which the waves will propagate is defined. The fourier transform of the object function is multiplied by the transfer function of the media and then inverse fourier transform is fianlly calculated.

\section{A. Transfer Functions Mathematics}

If medium 1 is filled with free space. The transfer function of free space $T_{1}\left(k_{x 1}\right)$ is [8]:

$T_{1}\left(k_{x 1}\right)=e^{-j \sqrt{\omega^{2} \varepsilon_{0} \mu_{0}-k_{x 1}^{2}} d_{1}}$

where $\varepsilon_{0}$ is the permittivity of medium $1, \mu_{0}$ is the permuability of medium 1 and $d_{1}$ is the horizontal distance of propagation in medium1.

The transfer function of medium2 (silver slab) $T_{2}\left(k_{x 2}\right)$ is [8]

$T_{2}\left(k_{x 2}\right)=\frac{4 \delta_{e}}{\left(1+\delta_{e}\right)^{2} e^{j k_{z 2} d_{2}}-\left(1-\delta_{e}\right)^{2} e^{-j k_{z 2} d_{2}}}$

where $d_{2}$ is the thickness of the slab which is inserted in medium 2 and $\delta_{e}$ is equal to:

$\delta_{e}=\frac{k_{z 2} \varepsilon_{0}}{k_{z 1} \varepsilon_{2}}$.

While medium 3 is filled with free space and the transfer function of free space $T_{3}\left(k_{x 3}\right)$ is [8]:

$T_{3}\left(k_{x 3}\right)=e^{-j \sqrt{\omega^{2} \varepsilon_{0} \mu_{0}-k_{x 3}^{2}} d_{3}}$

where $d_{3}$ is the horizontal distance of propagation in medium3.

The total transfer function for the whole three media is:

$T=T_{1}\left(k_{x 1}\right) * T_{2}\left(k_{x 2}\right) * T_{3}\left(k_{x 3}\right)$

In the next subsection, we apply equations 5-8 with the fourier transforms to an example to see the behaviour of the received image at the reciever line due to the presence of the hyperlens in medium 2.

\section{B. Transfer Functions Mathematics}

We investigate the ability of a silver hyperlens to produce images for an object that is much smaller than the wavelength used. An object of Aluminum rod of diameter $1 \mathrm{~cm}$ will be placed at the scattering plane as shown in fig. 1 (rod not shown). A wave with frequency $2 \mathrm{GHz}$ is used. A silver slab is placed in medium2. Silver is an epsilon-negative material with relative permittivity $\varepsilon_{r}=-1-0.01 j$. The width of the silver slab is $d_{2} \mathrm{~mm}$. The object will be placed at $d_{1} \mathrm{~mm}$ infront of the surface of the slab. We expect the image to appear at $d_{3}$ mm behind the rear surface of the slab. 

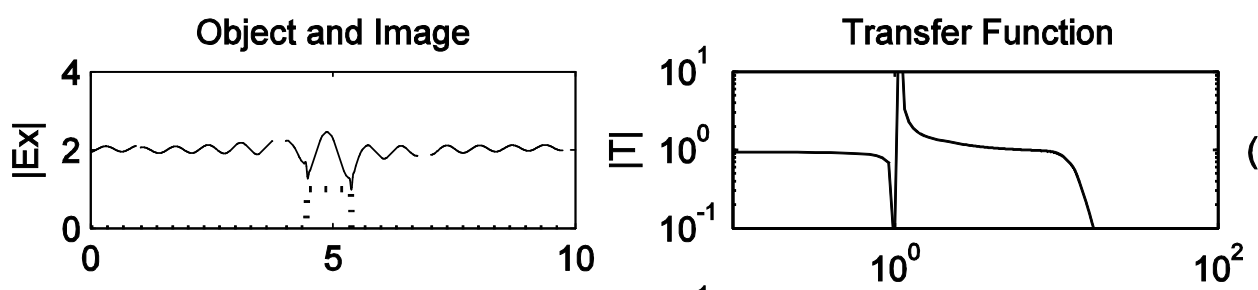

(a)
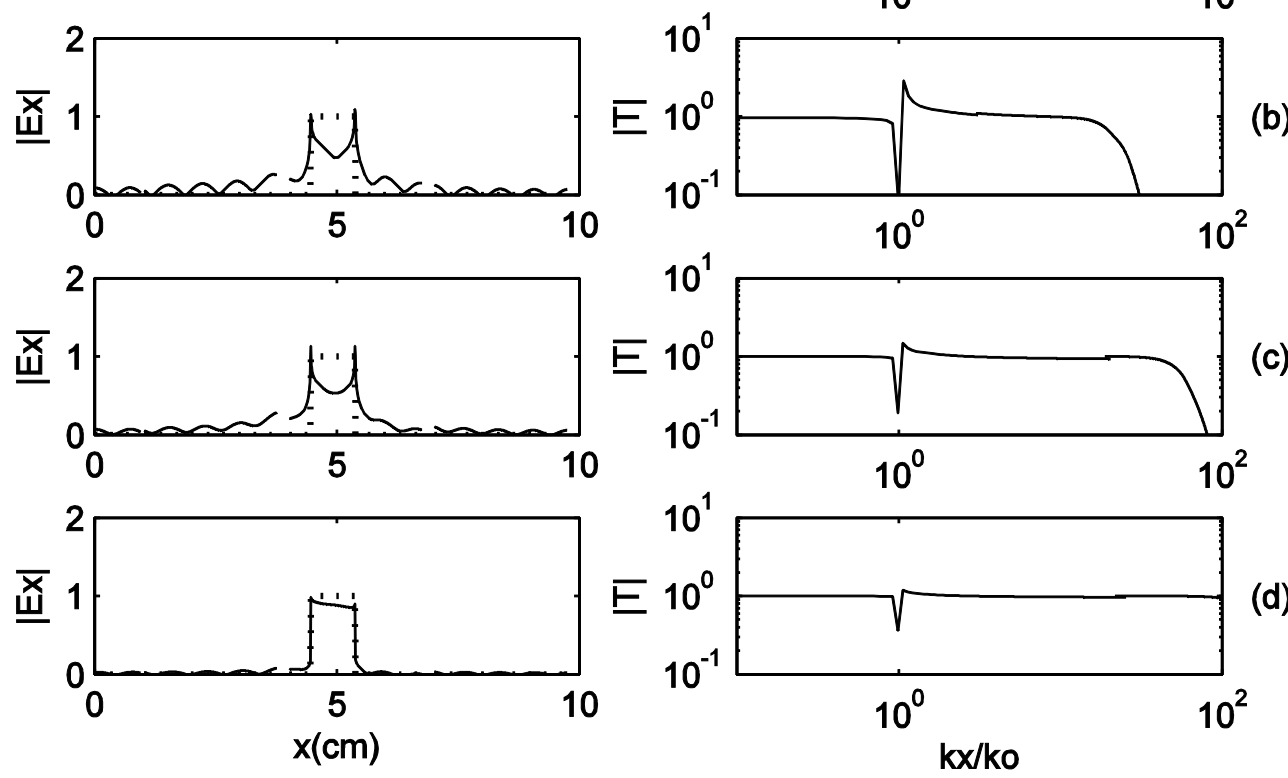

Figure 3: The plots on the left show the electric field (dotted lines) for an Aluminium rod which is placed at the scattering plane as shown in fig. 1. The solid lines represent the output electric field which is received at the receiver plane as shown in fig.1. The plots on the right show the amplitude of the transfer function Eq. 8. The thickness of the slab is $6,42,1 \mathrm{~mm}$ in the figures a-d consecutively.

For the first case, we put $d_{2}=6 \mathrm{~mm}$ and $d_{1}=d_{3}=3 \mathrm{~mm}$. In fig. 3a, we find in the plot on the right the amplitude of the transfer function $T$. For $k_{x}<\sqrt{\omega^{2} \varepsilon \mu},|T|$ is equal to one which means that all frequencies satisfying this equation will pass through the silver slab without attenuation. For $k_{x}>\sqrt{\omega^{2} \varepsilon \mu},|T|$ is slightly above one up till when $k_{x}$ is almost equal fifteen times $\sqrt{\omega^{2} \varepsilon \mu}$ then $|T|$ attenuates to almost zero. In the plot on the left, the amplitude of the electric field is drawn. The object (dotted line) is a step function of amplitude one and width $1 \mathrm{~cm}$. The image (solid line) is almost a line parallel to the $\mathrm{x}$-axis with amplitude equal two. The image doesn't represent the object being imaged. For the second case, we put $d_{2}=4 \mathrm{~mm}$ and $d_{1}=d_{3}=2 \mathrm{~mm}$. In fig. $3 \mathrm{~b}$, in the plot on the right $|T|$ is equal to one for all frequencies satisfying $k_{x}<\sqrt{\omega^{2} \varepsilon \mu}$. For $k_{x}>\sqrt{\omega^{2} \varepsilon \mu},|T|$ is almost equal to one up till when $k_{x}$ is almost equal twenty two times $\sqrt{\omega^{2} \varepsilon \mu}$ then $|T|$ attenuates to almost zero. In the plot on the left, the object (dotted line) is a step function of amplitude one and width $1 \mathrm{~cm}$. The image (solid line) has the shape of a Bessel function of the first kind with a maximum amplitude almost equal to one. The image is more representative of the object being imaged than in fig. 3a. For the third case, we put $d_{2}=2 \mathrm{~mm}$ and $d_{1}=d_{3}=1 \mathrm{~mm}$. In fig. $3 \mathrm{c}$, in the plot on the right $|T|$ is equal to one for all frequencies satisfying $k_{x}<\sqrt{\omega^{2} \varepsilon \mu}$. For $k_{x}>\sqrt{\omega^{2} \varepsilon \mu},|T|$ is almost equal to one up till when $k_{x}$ is almost equal fourty five times $\sqrt{\omega^{2} \varepsilon \mu}$ then $|T|$ attenuates to almost zero. In the plot on the left, the image (solid line) has the shape of a Bessel function of the first kind with the crests on both sides having lesser amplitude than those of fig $3 \mathrm{~b}$. The image is more representative of the object being 
imaged than that in fig. $3 \mathrm{~b}$. For the fourth case, we put $d_{2}=1 \mathrm{~mm}$ and $d_{1}=d_{3}=0.5 \mathrm{~mm}$. In fig. $3 \mathrm{~d}$, in the plot on the right $|T|$ is equal to one for all frequencies satisfying $k_{x}<\sqrt{\omega^{2} \varepsilon \mu}$. For $k_{x}>\sqrt{\omega^{2} \varepsilon \mu},|T|$ is slightly above one up till when $k_{x}$ is almost equal hundred times $\sqrt{\omega^{2} \varepsilon \mu}$ then $|T|$ attenuates to almost zero. In the plot on the left, the image (solid line) has almost the same shape of the object being imaged. The image is a good representatation of the object being imaged. As the thickness of the slab decreases, the range of evanescent frequencies that can be amplified through the slab increases. The evanescent waves captured at the receiver side holds detailed information about the object and so the obtained image is more perfect.

\section{DISCUSSION}

The behaviour of the electric field as it propagates in different media is firstly examined. As evanescent components are added to the propagated waves, a maxima is seen to move to the rear surface of the slab used. The amplitude of the fast decaying evanescent waves is shown to be amplified so that they are captured at the receiver side. The reason for the maxima that appear in all cases which are studied is the excitation of Surafce Plasmon Polaritons (SPP modes). The scattered waves incident on the surface of the slab excite the SPP at the rear side of the slab. These modes give the effect as if the amplitude is amplified or is increasing as waves propagate inside the slab. Secondly, the possibility of imaging an Aluminum rod by using a silver slab is investigated. The only case in which we got a good image representing the object being imaged is when we used a slab of thickness $1 \mathrm{~mm}$. As the thickness of the slab decreases, the range of evanescent frequencies that can be amplified through the slab increases. The evanescent waves captured at the receiver side holds detailed information about the object and so the accuracy of the calculated image improves. To obtain a perfect image for an object, the transfer function of the slab should be flat for all values of $k_{x}$. The value of the transfer function is almost one for an increasing range of evanescent components as the thickness of the slab decreases.

\section{CONCLUSION}

The quality of an image deteriorates due to the presence of evanescent waves which decays rapidly. The novelty here is the improvement of the resolution of the virtual confocal microscope method by several orders of magnitude and produce images of high contrast. The SPP plays an important part in amplifying the amplitude of the evanescent waves. The size of the silver slab is very important as well. The smaller the thickness of the slab the better the resolution of the captured images due to the increase in the cutoff frequency of the transfer function of the slab.

\section{REFERENCES}

[1] Hassanein, A. D., D. J. Edwards, et al. (27 to 30 October 2008). "UWB Tomography via Simulated Optical Systems". International Symposium on Antenna \& Propagation (ISAP08). Taipei, Taiwan.

[2] Hassanein A. M. D., Imaging Techniques Using Ultra Wide Band Waves, PhD Thesis, University of Oxford, UK. 2008.

[3] D. Schurig, J. B. Pendry and D. R. Smith, “Transformation designed optical elements”, Optical Society of America, 2007.

[4] B J Harker1, A D Chadwick, G L Harris. "ULTRA-WIDEBAND 3-DIMENSIONAL IMAGING". Roke Manor Research Limited, UK, 2008.

[5] Shouhei Kidera, Takuya Sakamoto, Toru Sato. "Accurate UWB Radar Three-Dimensional Imaging Algorithm for a Complex Boundary Without Range Point Connections.” IEEE TRANSACTIONS ON GEOSCIENCE AND REMOTE SENSING, VOL. 48, NO. 4, APRIL 2010.

[6] R.M. Narayanan, X. Xu, J.A. Henning. "Radar penetration imaging using ultra-wideband (UWB) random noise waveforms." IEE Proc.-Radar Sonar Navig., Vol. 151, No. 3, June 2004.

[7] Hecht, Eugene, 2002. Optics, 4th Ed, Addison Wesley.

[8] Solymar et al., 2009. Waves in Metamaterials. UK: Oxford. Oxford University Press. 